\title{
Bed-sore and Associated Factors Among Patients Admitted at Surgical Wards of Wolaita Sodo University Teaching and Referral Hospital, Southern Ethiopia
}

\author{
Aseb Arba*, Melese Meleku, Atinafu Nega, Esayas Aydiko \\ School of Nursing, College of Medicine and Health Sciences, Wolaita Sodo University, Wolaita Sodo, Ethiopia
}

Email address:

40aseb $@$ gmail.com (A. Arba)

${ }^{*}$ Corresponding author

\section{To cite this article:}

Aseb Arba, Melese Meleku, Atinafu Nega, Esayas Aydiko. Bed-sore and Associated Factors Among Patients Admitted at Surgical Wards of Wolaita Sodo University Teaching and Referral Hospital, Southern Ethiopia. American Journal of Clinical and Experimental Medicine.

Vol. 8, No. 4, 2020, pp. 56-62. doi: 10.11648/j.ajcem.20200804.11

Received: June 9, 2020; Accepted: June 19, 2020; Published: July 23, 2020

\begin{abstract}
Background: Bed sore has long been recognized as major cause of morbidity, mortality and health care burden globally and worst in developing countries like Ethiopia. It is one of key performance indicators of quality of care provided to patient. Therefore, this study was aimed to assess magnitude of bed sore and associated factors among patients admitted to surgical ward of Wolaita Sodo University Teaching and Referral Hospital, South Ethiopia. Method: We conducted institutionbased cross-sectional study from February- June, 2019. A systematic random sampling technique was applied to select 237 patients who were admitted to surgical ward of Hospital. We collected data through face to face interview using a structured and pretested questionnaire, prepared check lists and physical examination. After collection, we entered data into Statistical Package for Social Sciences Version 23. We conducted descriptive analysis and logistic regressions to identify factors associated with dependent variable. We declared presence of statistically significant association at p-value less than 0.05 at $95 \%$ confidence interval. Results: Eight patients or 3.4\% among 237 admitted patients developed bedsore. Patients older than fifty three years $(\mathrm{AOR}=11.84,95 \% \mathrm{CI}(1.05,133.259)$, living in rural area $(\mathrm{AOR}=6.64,95 \% \mathrm{CI}(1.09,40.58)$ and being bedridden patient $(\mathrm{AOR}=7.82,95 \% \mathrm{CI}(1.27,48.38)$ possessed factors associated with bedsore development. Conclusion and recommendation: The magnitude of bed sore was medium; but it needs improvement because it is one of criteria that indicate quality of service rendered in hospital. Bedsore occurrence was associated with increasing age, rural residence and being bedridden patient. Therefore, frequent positioning of the patient and health education for patients from rural areas and special and frequent care for those unable to care for themselves were needed to minimize occurrence of bed sore.
\end{abstract}

Keywords: Pressure Ulcer, Bed Sore, Decubitus Ulcer, Bedridden Patient

\section{Introduction}

Bed sore is severe condition of skin due to localized injury on the skin and/or underlying tissue usually over a bony prominence area. Bed sore is one of the most common complications for bed-ridden patients hospitalized in acute and chronic care services and imposes a major burden on patients, their relatives and caregivers. Loss of sensory perception and immobility are the main risk factors for decubitus ulcers because patients may not be aware of the discomfort and do not change their position to relieve the pressure [1-6].

Bed sores are a serious and common problem for older persons, affecting approximately 1 million adults in the United States. As the population ages, pressure ulcers will continue as a major health care problem. Local blood supply to the skin decreases, epithelial layers flatten and thin, subcutaneous fat decreases, and collagen fibers lose elasticity. The prevention and healing of pressure ulcers requires the cooperation and skills of the entire interdisciplinary health care team [7-1].

The frequent assessment and effective skin care prevent the development of bed sore. Nurses should develop management plan to prevent development of bed sore in patients who are identified as being at risk. It improves 
healing and prevents complications of existing pressure ulcer. This is because nurses have a responsibility to monitor patient's skin in order to plan, implement and evaluate interventions that maintain skin integrity by using their knowledge and skill [3, 13-17].

Bed sore is a serious condition that needs cooperation among all the medical disciplines. Pressure ulcers are serious and common health concern affecting about 1.5-3 million adults and is a serious health concern among elders. Despite advances in medical technology and the use of formal prevention programs based on clinical practice guidelines, the prevalence of bed sore during hospitalization continues to increase to $80 \%$. The study finding on prevalence and associated factors of pressure ulcer among hospitalized patients in Felege Hiwot referral hospital revealed that $16.8 \%$ of them had pressure ulcer in Ethiopia [2, 13, 15].

Bed sore is serious condition that leads to ischemia, cell death, and tissue necrosis, as capillaries are compressed and the blood flow is restricted. The cutaneous tissues become broken or destroyed, leading to progressive destruction and necrosis of underlying soft tissues. Several factors were contributed to pressure ulcer development. These were intrinsic factors such as advanced age ( $>65$ years), limited mobility, poor nutrition, co-morbidities, incontinence impaired sensory perception, altered tissue perfusion, organ system failure, and infection and extrinsic factors like force and duration of pressure, shearing, friction and macerations [22-24].

Though modern medical science has launched a variety of treatment methods, prevention is the best way to get rid of it. Bed sores are best prevented by using support surfaces that are volatile in nature-like water mattress, variable pressure mattress $[2,10,15,20]$.

Although there are some pressure ulcer prevention protocols in public hospitals, still there is number of patients suffering from bed sore and it is serious condition that spreads across all the medical disciplines and considerable health care problem. Therefore, it important to assess magnitude of bed sore and its associated factors among patients admitted at Wolaita Sodo University Teaching and Referral Hospital in Surgical Ward South Ethiopia.

Objectives

1. To determine magnitude of bed sore among patients admitted at surgical ward of Wolaita Sodo University Teaching and Referral Hospital, 2019.

2. To identify factors associated with bed sore among patients admitted at surgical ward of Wolaita Sodo University Teaching and Referral Hospital, 2019.

\section{Methods and Materials}

\subsection{Study Setting and Population}

We conducted a hospital-based cross-sectional study in Wolaita Sodo University teaching and referral Hospital (WSUTRH) from February- June, 2019. This hospital is found in Wolaita Sodo Town. WSUTRH has about 300 beds and 5 operation rooms. All patients admitted to WSUTRH were considered as source population. We randomly selected study population from patients admitted to surgical wards during the study period.

\subsection{Inclusion and Exclusion Criteria}

All patients admitted to surgical wards of hospital for more than two days before data collection were included in the study. Patients whose age less than 18 years old, unable to sign consent, mentally ill and patients who are admitted for the second time during data collection period were excluded from study.

\subsection{Sample Size and Sampling Technique}

The sample size was determined using single population proportion formula. The sample size was calculated taking prevalence of pressure ulcer among hospitalized patients in Felege Hiwot Referral Hospital $(\mathrm{P}=16.8 \%)$ [15]. The final sample size after adding $10 \%$ non-response rate was 237 .

We got study participants by using systematic random sampling technique. The total number of study participants was proportionally distributed to different units of surgical ward according to number of patients admitted.

\subsection{Data Collection Tools and Procedures}

The data was collected through face to face interview using structured questionnaire and physical examination. The Braden scale risk assessment tool was used to assess the risk of pressure ulcer or bed sore and comprised the following items: sensory perception, skin moisture, physical activity, mobility, nutrition, and friction/shear [40]. The standardized pre-tested structured questionnaire which prepared in English was used.

Data was collected by two nurses and supervised by one senior nurse. Brief training and pretest was done before actual data collection. Data was checked daily for completeness and quality by supervisor.

\subsection{Study Variables}

\subsubsection{Dependent Variable}

Bed sore.

\subsubsection{Independent Variables}

Socio-demographic, economic variables: age, sex, residence, marital status, educational status, and monthly income.

Braden Risk Assessment Scale (RAS) factors; sensory perception, moisture, activity, friction /shear, mobility and nutrition.

Health facility related and other factors: length of hospital stay, supportive device, position change, bed ridden and BMI \& Smoking.

\subsubsection{Operation Definitions and Terms of Definition}

Bed sore: any lesion caused by unrelieved pressure resulting in damage of skin and underlying tissue of admitted patients.

\subsubsection{Data Management and Analysis}

Data was coded and entered into to the Statistical Package 
for Social Science (SPSS) version 20 and analysis was done. The data was presented in tables, graphs, and figures. Bivariate analysis was done and we selected those variables with p-value less than or equal to 0.25 to multivariate analysis. $\mathrm{P}<0.05$ and AOR at $95 \%$ confidence interval were used to assess presence and strength of association between independent variable and dependent variable respectively.

\subsubsection{Ethical Consideration}

Ethical approval was obtained from research and ethical review committee of Wolaita Sodo University. Permission letter was given to WSUTRH administrations. We gave adequate information to all participants regarding the purpose, risk and benefit, and confidentiality of the study as described in the information. Participation was fully voluntary and informed written consent was obtained from each participant. This study was conducted according to declaration of Helsinki.

\section{Results}

\subsection{Socio-Economic Characteristics}

A total of 235 admitted patients to surgical ward of Wolaita Sodo University teaching and referral hospital participated in this study with the response rate of $99.15 \%$. The study participants (aged 18-59 years, with the mean $( \pm$ SD) age of $40.03( \pm 12.276)$ were interviewed. Among study participants, $151(64.3 \%)$ were male and 154 (65.5\%) were urban dwellers. 57 (24.3\%) of participants didn't attend any formal education, while only $11(4.7 \%)$ had college and above education. $194(82.6 \%)$ of the study participants were married and $97(41.3 \%)$ were farmer. The category of average monthly income of the participants, $126(53.6 \%)$ earned between $<1500$ ETB, and 109 (46.4\%) earned more than 1500 ETB shown in table 1.

Table 1. Socio - Demographic and Economic characteristics among patients admitted at Surgical Ward of WSUTRH, Southern Ethiopia, 2019 ( $n=235)$.

\begin{tabular}{llll}
\hline Variable & Category & No. & \% \\
\hline \multirow{4}{*}{ Age in year } & $18-32$ & 118 & $(50.2)$ \\
& $33-53$ & 87 & $(37.0)$ \\
Sex of the respondent & $\geq 54$ & 30 & $(12.8)$ \\
& Male & 151 & $(64.3)$ \\
& Female & 84 & $(35.7)$ \\
Residence & Urban & 154 & $(65.5)$ \\
& Rural & 81 & $(34.5)$ \\
& No formal education & 57 & $(24.3)$ \\
Educational status & Read \& write & 48 & $(20.4)$ \\
& Elementary & 71 & $(30.2)$ \\
& High school & 48 & $(20.4)$ \\
& College and above & 11 & $(4.7)$ \\
marital status & Single & 23 & $(9.8)$ \\
& Married & 194 & $(82.6)$ \\
& Divorced & 7 & $(3.0)$ \\
& Widowed & 11 & $(4.7)$ \\
& Employed & 33 & $(14.0)$ \\
Occupational status & Self employed & 34 & $(14.5)$ \\
& Not actively working yet & 6 & $(2.6)$ \\
& Merchant & 65 & $(27.7)$ \\
Monthly income in & Farmer & 97 & $(41.3)$ \\
Ethiopian Birr & $<1500$ & 126 & $(53.6)$ \\
& $\geq 1500$ & 109 & $(46.4)$ \\
\hline
\end{tabular}

\subsection{Disease and Health Facility Related and Other Factors}

The main reasons for admission among study participants during study period were urinary incontinence in $64(27.2 \%)$, road traffic accidents in $38(16.2 \%)$ and cancer in 29 $(12.3 \%)$. While, $22(9.4 \%)$ were admitted to diabetes with infections and head injury in figure 1 .

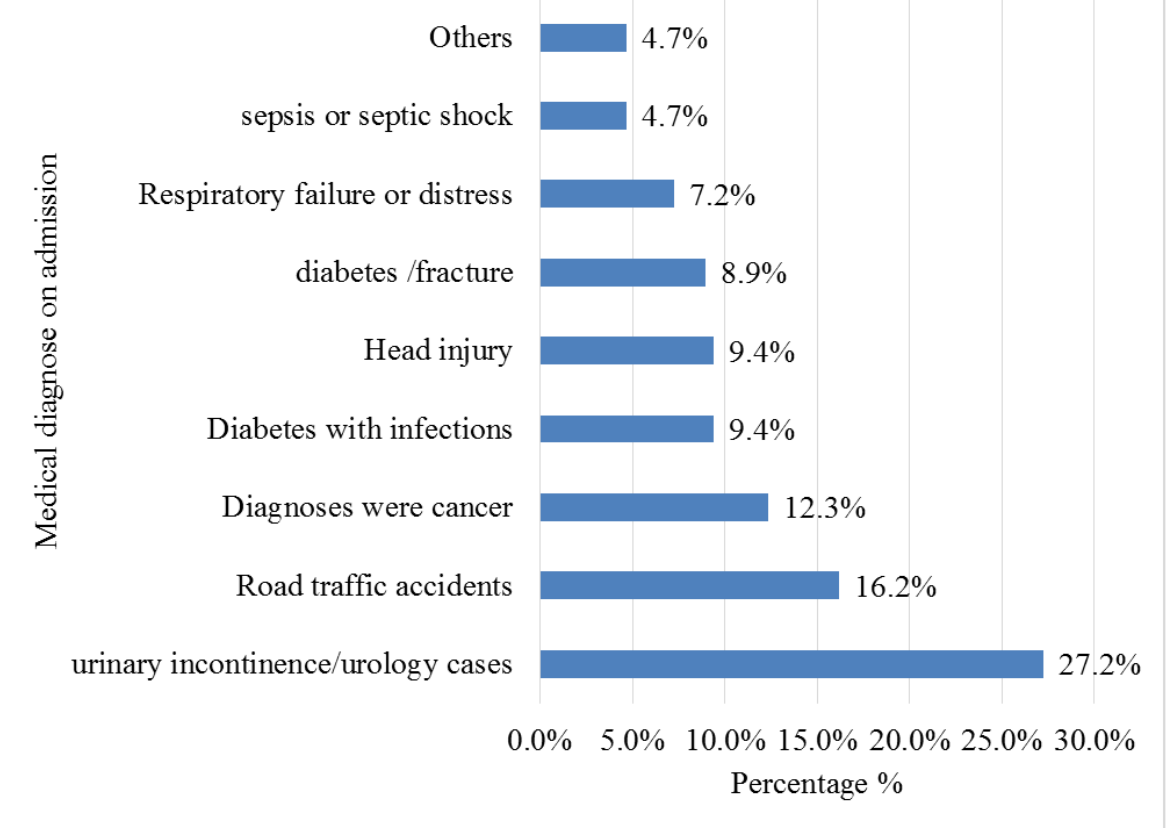

Figure 1. Medical diagnoses of patients admitted at Wolaita Sodo University Teaching and Referral Hospital in surgical ward, 2019. 
Out of the 235 participants admitted at surgical ward, 6 $(2.6 \%)$ and $8(3.4 \%)$ were currently smoking and smoked previously, respectively. One third of the participants 70 $(29.8 \%)$ were bedridden patients. Majority of the study participants $230(97.9 \%)$ reported they were turned 4 times/day shown in table 2 .

Table 2. Health facility related and other factors among patients admitted at surgical ward of WSUTRH, Southern Ethiopia, 2019 (n=235).

\begin{tabular}{llll}
\hline Variable & Category & No. & \% \\
\hline \multirow{3}{*}{ Smoke Cigarette } & Currently smoking & 6 & $(2.6)$ \\
& Previously smoke & 8 & $(3.4)$ \\
& No smoke & 221 & $(94.0)$ \\
The patient bedridden & Yes & 70 & $(29.8)$ \\
The patient gets position & Yo & 165 & $(70.2)$ \\
change service & No & 87 & $(37.0)$ \\
How often the patient & Every 2 to 3 hours & 3 & $(1.3)$ \\
gets position change & Every 4 hours & 2 & $(.9)$ \\
service & Turned 4times/day & 230 & $(97.9)$ \\
\hline
\end{tabular}

Regarding to the BMI, 166 (70.6\%) had normal weight. While, $27(11.5 \%)$ were Underweight, $36 \quad(15.3 \%)$ overweight and $6(2.6 \%)$ were obese shown in figure 2 .

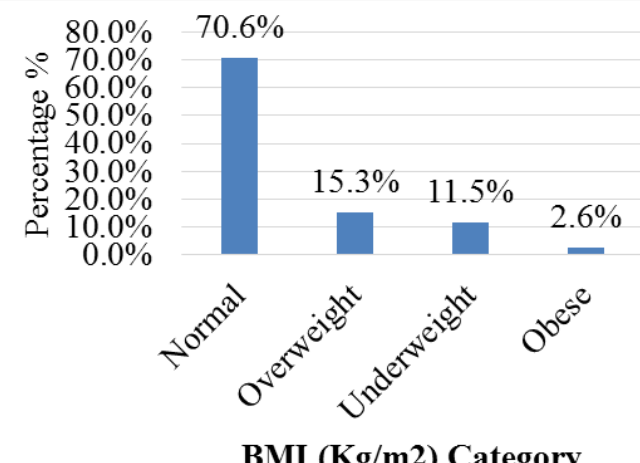

Figure 2. The nutritional status of the patients admitted in surgical ward at WSUTRH in Surgical Ward, Southern Ethiopia, 2019 (n=235).

\subsection{Magnitude of Bedsore}

The Magnitude of bed sore among patients admitted at surgical ward of Wolaita Sodo University Teaching and Referral Hospital during study period was 8 (3.4\%), 95\%CI: $(1.6,6.3)$ as shown in figure 3 .

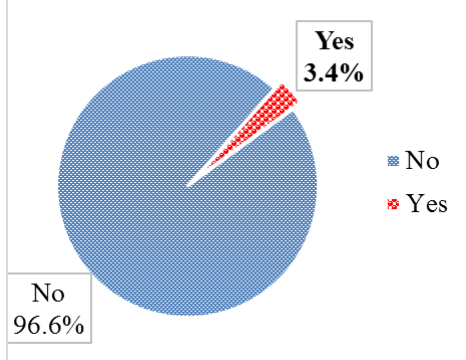

Figure 3. The prevalence of bed sore among patients admitted at WSUTRH in Surgical Ward, Southern Ethiopia, $2019(n=235)$.
Out of the $8(3.4 \%)$ who had developed bedsore, half of the participants $4(50.0 \%)$ were stage I and mainly affected anatomical location of pressure ulcer was $4(50.0 \%)$ were Sacral. The length of stay reported was mainly $\leq 7$ days and 8-15 days shown in table 3 below.

Table 3. Bed sore related factors among patients admitted at Surgical Ward of WSUTRH, Southern Ethiopia, $2019(n=8)$.

\begin{tabular}{llll}
\hline Variable & Category & No. & \% \\
\hline \multirow{4}{*}{ The stage of } & Stage I & 4 & $(50.0)$ \\
bedsore & Stage II & 1 & $(12.5)$ \\
& Stage III. & 1 & $(12.5)$ \\
& Stage IV & 1 & $(12.5)$ \\
The anatomical & Unstageable & 1 & $(12.5)$ \\
location of & Sacral & 4 & $(50.0)$ \\
bedsore & Shoulder & 1 & $(12.5)$ \\
& Elbow & 1 & $(12.5)$ \\
\multirow{2}{*}{ Length of stay } & Other & 1 & $(12.5)$ \\
& $\leq 7$ days & 1 & $(12.5)$ \\
& 8-15 days & 3 & $(37.5)$ \\
& $\geq 16$ days & 3 & $(37.5)$ \\
\hline
\end{tabular}

\subsection{Braden Risk Assessment Scale (RAS)}

Among 8 (3.4\%) patients who had developed pressure ulcers or bedsore, no complete impairment in sensory perception was reported, while, $4(50.0 \%)$ participants reported slight limitation and $3(37.5 \%)$ were very limited. Concerning moisture, 2 (25.0\%), $3(37.5 \%)$ and $2(25.0 \%)$ were very moist, occasionally moist and rarely moist respectively. Patients with bedsore, 4 (50.0\%) walked occasionally, $4(50.0 \%)$ were probably due to inadequate nutrition and $5(62.5 \%)$ reported friction and shear as cause of bed sore development shown in table 4 .

Table 4. Braden Risk Assessment Scale (RAS) factors among patients admitted at Surgical Ward of WSUTRH, Southern Ethiopia, 2019 (n=8).

\begin{tabular}{llll}
\hline Variable & Category & No. & \% \\
\hline \multirow{3}{*}{ Sensory perception } & Very limited & 3 & $(37.5)$ \\
& Slightly limited & 4 & $(50.0)$ \\
& No impairment & 1 & $(12.5)$ \\
& constantly moist & 1 & $(12.5)$ \\
Moisture & Very moist & 2 & $(25.0)$ \\
& occasionally moist & 3 & $(37.5)$ \\
& rarely moist & 2 & $(25.0)$ \\
Activity & Bedfast & 1 & $(12.5)$ \\
& chair fast & 2 & $(25.0)$ \\
& walks occasionally & 4 & $(50.0)$ \\
Mobility & walks frequently & 1 & $(12.5)$ \\
& Very limited & 3 & $(37.5)$ \\
& slightly limited & 3 & $(37.5)$ \\
Nutrition & No limitation & 2 & $(25.0)$ \\
& Very poor & 3 & $(37.5)$ \\
Friction and shear & probably inadequate & 4 & $(50.0)$ \\
& Adequate & 1 & $(12.5)$ \\
& Problem & 3 & $(37.5)$ \\
& potential problem & 5 & $(62.5)$ \\
\hline
\end{tabular}

\subsection{Factors Associated with Bedsore Among Admitted Patients}

Variables whose p-value less than 0.25 became candidate for 
multivariate analysis. In bivariate analysis, age in year, monthly income in Ethiopian birr, residence, educational status, the patient bedridden and the patient get position change service became candidate for multivariate analysis. In multivariate analysis, age in year, residence and the patient bedridden were variables with p-value of less than 0.05 . Specifically, patients aged 54 and above years had significant association with the prevalence of bedsore with had
$\mathrm{AOR}=11.843$ at 95 CI $(1.052,133.259)$. The study participants who were from rural area were more likely to develop bedsore when compared with urban dwellers $(\mathrm{AOR}=6.639$ at $95 \mathrm{CI}(1.09,40.58))$. Being the bedridden patient had significant association with the prevalence of bedsore $(\mathrm{AOR}=7.82$ at $95 \mathrm{CI}(1.265,48.377)$ as described in table 5 .

Table 5. Bivariate and multivariate analysis result of prevalence of bedsore and associated factors among patients admitted at Surgical Ward of WSUTRH, Southern Ethiopia, 2019.

\begin{tabular}{|c|c|c|c|c|c|c|}
\hline \multirow{2}{*}{ Variables } & \multirow{2}{*}{ Category } & \multicolumn{2}{|c|}{ Bedsore } & \multirow{2}{*}{ COR $(95 \% C I)$} & \multirow{2}{*}{$\operatorname{AOR}(95 \% C I)$} & \multirow{2}{*}{ P-Value } \\
\hline & & Yes & No & & & \\
\hline \multirow{3}{*}{ Age (years) } & $18-32$ & 2 & 116 & 1 & 1 & \\
\hline & $33-53$ & 3 & 84 & $2.07(0.34,12.67)$ & $2.88(0.39,21.20)$ & 0.299 \\
\hline & $\geq 54$ & 3 & 27 & $6.44(1.03,40.48)$ & $11.84(1.05,13.26)$ & $0.045^{*}$ \\
\hline \multirow{2}{*}{ Income (Eth. Birr) } & $<1500$ & 6 & 120 & $2.68(0.53,13.54)$ & $1.73(0.27,11.22)$ & 0.565 \\
\hline & $\geq 1500$ & 2 & 107 & 1 & 1 & \\
\hline Residence & Urban & 3 & 151 & 1 & 1 & \\
\hline \multirow{5}{*}{ Educational status } & Illiterate & 1 & 56 & 1 & 1 & \\
\hline & Read \& write & 1 & 47 & $1.19(0.07,19.57)$ & $1.22(0.06,23.68)$ & 0.90 \\
\hline & Elementary & 2 & 69 & $1.62(0.14,18.37)$ & $2.015(0.16,25.44)$ & 0.588 \\
\hline & High school & 2 & 46 & $2.44(0.21,27.71)$ & $4.86(0.31,76.65)$ & 0.261 \\
\hline & College and above & 2 & 9 & $12.44(1.02,151.84)$ & $7.14(0.38,135.09)$ & 0.191 \\
\hline \multirow{2}{*}{ Bedridden } & No & 2 & 163 & 1 & 1 & \\
\hline & Yes & 6 & 64 & $7.64(1.50,38.85)$ & $7.82(1.27,48.38)$ & $0.027^{*}$ \\
\hline
\end{tabular}

\section{Discussion}

The magnitude of bed sore among patients admitted at surgical ward of Wolaita Sodo university teaching and referral hospital during study period was $8(3.4 \%), 95 \% \mathrm{CI}$ $(1.6,6.3)$. This finding goes in line with study conducted in the prevalence of bed sore among patients admitted was 3.4\% at Debre Markos Referral Hospital, East Gojam Zone, Ethiopia [37].

The magnitude of bedsore in this study was lower than studies done in in Brazil [31], Turkey [32], and Germany [33] with magnitude of $12.7 \%, 10.4 \%$, and $11.7 \%$, respectively. The magnitude of bed sore was also lower than the study conducted in Sweden (22.9\%) [34], Italy (27\%)[35], Thailand (47.6\%) [36], and Felege Hiwot referral hospital Ethiopia (16.8\%)[15]. This difference might be due to increasing number of aging population that admits for long-term care in developed countries. In addition, this might be due to different characteristics of participants, disease condition of patients and length of stay in hospital.

Concerning factors, patients aged 54 and above had significant association with the occurrence of bedsore when compared with participants who are younger in age. This finding is similar with the study report on age showed a significant association with prevalence of bed sore in King Abdulaziz University Hospital [23], Atlantic salmon [24], [39]. This might be due to advancing in age may limited mobility, cause poor nutrition, accompanying co-morbidities, incontinence, impaired sensory perception, altered tissue perfusion, organ system failure, and infection and extrinsic factors like force and duration of pressure, shearing, friction and macerations. Addition to that, it may due to advancing in age of participants may expose to the bedsore because of decreased functioning of nervous system.

The study participants who live in rural area were six times more likely to develop bedsore when compared with urban dwellers. This study result is similar with the study finding in Thailand Thai stroke patients [36]. They might lack full information and advice about care of dependent patients and prevention of bedsore development.

The odds of occurrence of bedsore were high among bedridden patient when compared with their counterparts. This study finding was similar to the finding of the study conducted in Debre Markos Referral Hospital, East Gojjam Zone, Ethiopia [37] and conducted in Jimma University Medical Center, Southwestern Ethiopia [38]. This might be due to their inability to care for themselves and staying in the same position for longtime which causes compression of blood vessels, nerve endings and damage skin tissues.

\section{Conclusion and Recommendation}

The magnitude of bed sore among patients admitted at hospital in surgical ward was medium. Advancing of age, rural residence and being bedridden patient were factors associated with the occurrence of bed sore. Therefore, frequent positioning of the patient and health education for patients from rural areas and special and frequent care for those unable to care for themselves were needed to minimize occurrence of bed sore. Future studies shall consider qualitative methods. 


\section{Ethics Approval and Consent to Participate}

This study was conducted in accordance with declaration of Helsinki that provides guidance for researcher to protect research subjects. Study was approved by Institutional research review committee of Wolaita Sodo University. Then, the participants were informed as the participation is voluntary basis and about benefits and harms of participation. Informed written consent is obtained from participants before data collection.

\section{Consent to Publish}

All Authors agreed to public this original research work.

\section{Conflicts of Interest}

The authors declare that they have no competing interests.

\section{Acknowledgements}

In addition, Authors would like to acknowledge officials at different level and participants who gave response without restriction.

\section{References}

[1] Bates-Jensen BM., McCreath HE., Harputlu D., Patlan A. Reliability of the Bates-Jensenn Wound Assessment Tool (BWAT) for Pressure Injury Assessment: The Pressure Ulcer Detection Study. Wound repair and regeneration: official publication of the Wound Healing Society [and] the European Tissue Repair Society. 2019.

[2] Kandha Vadivu P. Design and Development of Portable Support Surface and Multilayered Fabric Cover for Bed Sore Prevention. The Indian journal of surgery. 2015; 77 (Suppl 2): 576-82.

[3] Nakanishi T. A report on a clinical experience of which has successfully made several antibiotics-resistant bacteria (MRSA etc.) negative on a bedsore. 1993 Sep; 63 (3): 95100 .

[4] Wang Q., Qian Z., Liu B., Liu J., Zhang L. In vitro and in vivo evaluation of new PRP antibacterial moisturizing dressings for infectious wound repair. Journal of biomaterials science Polymer edition. 2019: 1-20.

[5] Kimura T. Physiopathology, prevention and therapy of decubitus ulcer. Cases of refractory decubitus ulcer giant before and multiple bedsore]. Kangogaku zasshi. 2005; 39 (10): 1029-32.

[6] Li Y, Zhu C, Fan D, Fu R, Ma P, et al. A Bi-Layer PVA/CMC/PEG Hydrogel with Gradually Changing Pore Sizes for Wound Dressing. Macromolecular bioscience. 2019: e1800424.

[7] Piotrowski S., Wilson L., Dharmaraj N., Hamze A., Clark A., et al. Development and Characterization of a Rabbit Model of Compromised Maxillofacial Wound Healing. Tissue engineering Part C, Methods. 2019.

[8] Jahne-Raden N., Kulau U., Marschollek M., Wolf K H. INBED: A Highly Specialized System for Bed-Exit-Detection and Fall Prevention on a Geriatric Ward. Sensors. 2019; 19 (5).

[9] Jimenez-Garcia JF., Arboledas-Bellon J., Ruiz-Fernandez C., Gutierrez-Garcia M., Lafuente-Robles N., et al. The advanced practice nurse in the adequacy of chronic, complex wound care. Enfermeria clinica. 2019.

[10] Sedmak D., Vrhovec M., Huljev D. Prevention of pressure ulcer (bedsore). Acta medica Croatica: casopis Hravatske akademije medicinskih znanosti. 2013; 67 Suppl 1: 29-34.

[11] Leyva-Moral J M. How to treat a bed sore with a tendon exposed. Revista de enfermeria. 2009; 32 (1): 25-8.

[12] Preece J. Total bed management: the way forward in pressure sore prevention. British journal of nursing. 2009; 8 (22): 1524-6, 8-9.

[13] Komakech R, Matsabisa M G, Kang Y. The Wound Healing Potential of Aspilia africana (Pers.) C. D. Adams (Asteraceae). Evidence-based complementary and alternative medicine: eCAM. 2019; 2019: 7957860.

[14] Bakaeen FG., Haddad O., Ibrahim M., Pasadyn SR., Germano E., et al. Advances in managing the noninfected open chest after cardiac surgery: Negative-pressure wound therapy. The Journal of thoracic and cardiovascular surgery. 2018.

[15] Haileyesus G., Mignote H., Abdella A. Prevalence and Associated Factors of Pressure Ulcer among Hospitalized Patients at Felegehiwot Referral Hospital, Bahir Dar, Ethiopia. Hindawi Publishing Corporation, Advances in Nursing. 2014; 14 (1): 8.

[16] Li J., Guo XP., Wang KH., Zhao DH., Han T., et al. Closure of cystic cavity-type bedsore by subcutaneous undermining dissection with continuous negative pressure drainage. Zhonghua zheng xing wai ke za zhi Zhonghua zhengxing waike zazhi Chinese journal of plastic surgery. 2012; 28 (2): 113-5.

[17] Acker G., Schlinkmann N., Fekonja L., Grunwald L., Hardt J., et al. Wound healing complications after revascularization for moyamoya vasculopathy with reference to different skin incisions. Neurosurgical focus. 2019; 46 (2): E12.

[18] Adegoke BOA., Odole AC., Akindele LO., Akinpelu AO. Pressure ulcer prevalence among hospitalised adults in university hospitals in South-west Nigeria. wound Pract Pesearch. 2013; 21 (2): 128-34.

[19] Gu A., Malahias MA., Strigelli V., Nocon AA., Sculco TP., et al. Preoperative Malnutrition Negatively Correlates With Postoperative Wound Complications and Infection After Total Joint Arthroplasty: A Systematic Review and Meta-Analysis. The Journal of arthroplasty. 2019.

[20] Zhao Z., Zhang B., Liu Y., Zhang R., Liang Q., et al. Repair of bedsore over greater trochanter in paraplegic patients with rectus femoris island myocutaneous flap. Zhonghua shao shang za zhi Zhonghua shaoshang zazhi Chinese journal of burns. 2014; 30 (3): 227-30.

[21] Pasichniy D A. a Sacral Bedsore Plasty, Using Rotational Cutaneo-Fascial Gluteal Flaps. Klinichna khirurhiia. 2015 (10): 57-60. 
[22] Hampton S. Bedsore neglect is a result of reduced education and resources. British journal of nursing. 2011; 20 (15): S27.

[23] Albishi W., Albeshri MA., Mortada HH., Alzahrani K., Alharbi R., et al. Awareness and Level of Knowledge About Surgical Site Infections and Risks of Wound Infection Among Medical Physicians in King Abdulaziz University Hospital: Cross-Sectional Study. Interactive journal of medical research. 2019; 8 (1): e12769.

[24] Sveen LR., Timmerhaus G., Krasnov A., Takle H., Handeland S., et al. Wound healing in post-smolt Atlantic salmon. Scientific reports. 2019; 9 (1): 3565.

[25] Varela P., Sartori S., Viebahn R., Salber J., Ciardelli G. Macrophage immunomodulation: An indispensable tool to evaluate the performance of wound dressing biomaterials. Journal of applied biomaterials \& functional materials. 2019; 17 (1).

[26] Kielo E., Salminen L., Suhonen R., Puukka P., Stolt M. Graduating student nurses' and student podiatrists' wound care competence: a cross-sectional study. Journal of wound care. 2019; 28 (3): 136-45.

[27] Ji QY., Wang M., Li W. Nutrition Support is a Potential Confounding Factor for the Relationship Between Body Weight Variation and the Risk of Bedsore. Critical care medicine. 2019; 47 (1): 64.

[28] Mariam AL., El-Sehsah EM., Ramadan MA. Risk factors and distribution of MDROs among patients with healthcare associated burn wound infection. Germs. 2018; 8 (4): 199206.

[29] Wallen TJ., Habertheuer A., Gottret JP., Kramer M., Abbas Z, et al. Sternal wound complications in patients undergoing orthotopic heart transplantation. Journal of cardiac surgery. 2019.

[30] Svensson-Bjork R., Zarrouk M., Asciutto G., Hasselmann J., Acosta S.. Meta-analysis of negative pressure wound therapy of closed groin incisions in arterial surgery. The British journal of surgery. 2019; 106 (4): 310-8.

[31] Juleita MF., Leila B., Bernando H., Lydia MF. "Prevalence of pressure ulcers among the elderly living in longstay institutions in Sao Paulo," Sao Paulo Medical Journal, vol. 2009; 127 (4): 211-5.

[32] Oztunc G., Inan DG. "Pressure ulcer prevalence in Turkey: a sample from a university hospital," Journal of Wound, Ostomy and Continence Nursing. 2012; 39 (4): 409-13.

[33] Lahmann NA., Halfens RJ., Dassen T. "Prevalence of pressure ulcers in Germany," Journal of clinical nursing. 2005; 14 (2): 165-72.

[34] Gunningberg L., Stotts NA. "Tracking quality over time: what do pressure ulcer data show?" International Journal for Quality in Health Care. 2008.; 20 (4): 246-53.

[35] Capon A., Pavoni N., Mastromattei A., Di Lallo D. "Pressure ulcer risk in long-term units: prevalence and associated factors,". Journal of Advanced Nursing. 2007; 58 (3): 263-72.

[36] Suttipong C., and Sindhu S. "Predicting factors of pressure ulcers in older Thai stroke patients living in urban communities," Journal of clinical nursing. 2012; 21 (4): 372 9.

[37] Benalfew L., Balcha B., Desalegn H., Bekele T. Prevalence and Associated Factors of Pressure Ulcer among Hospitalized Adults at Debre Markos Referral Hospital, East Gojjam Zone, Ethiopia. Addis Ababa University College of Health Sciences 2016.

[38] Assefa T, Mamo F, Shiferaw D. Prevalence of Bed Sore and its associated Factors among Patients admitted at Jimma University Medical Center, Jimma Zone, Southwestern Ethiopia, 2017 Cross-sectional study. Ortho \& Rheum Open Access. 2017; 8 (4).

[39] Probst S., Holloway S., Rowan S., Pokorna A. Wound Curriculum for Nurses: Post-registration qualification wound management-European qualification framework level 6 . Journal of wound care. 2019; 28: S1-S33.

[40] Barbara Braden, Nancy Bergstrom. Braden Scale for Predicting Pressure Ulcer Risk. 2004; 1992 (3). 